\title{
Fluorescence of the Flavin group in choline oxidase. Insights and analytical applications for the determination of choline and betaine aldehyde.
}

E. Ortegaa , S. de Marcosa, I. Sanz-Vicentea, C. Ubideb, M. Ostrab, M.Vidalb and J. Galbána, ${ }^{*}$.

${ }^{a}$ Analytical Biosensors Groups, Analytical Chemistry Department, Faculty of Sciences, Aragon Institute of Nanoscience, University of Zaragoza, 50009-Zaragoza, Spain.

'Departamento de Química Aplicada, Facultad de Química, Universidad del País Vasco, Manuel de Lardizábal 3, 20018-San Sebastián, Spain.

*Corresponding author: e-mail: jgalban@unizar.es; Phone: +34976761291

\section{Abstract}

Choline oxidase $(\mathrm{ChOx})$ is a flavoenzyme catalysing the oxidation of choline $(\mathrm{Ch})$ to betaine aldehyde (BA) and glycine betaine (GB).

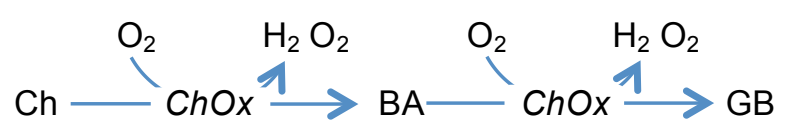

In this paper a fundamental study of the intrinsic fluorescence properties of ChOx due to Flavin Adenine Dinucleotide (FAD) is presented and some analytical applications are studied in detail. Firstly, an unusual alteration in the excitation spectra, in comparison with the absorption spectra, has been observed as a function of the $\mathrm{pH}$. This is ascribed to a change of polarity in the excited state. Secondly, the evolution of the fluorescence spectra during the reaction seems to indicate that the reaction takes place in two consecutive, but partially overlapped, steps and each of them follows a different mechanism. Thirdly, the chemical system can be used to determine the $\mathrm{Ch}$ concentration in the range from $510^{-6} \mathrm{M}$ to $510^{-5} \mathrm{M}$ (univariate and multivariate calibration) in the presence of BA as interference, and the joint $\mathrm{Ch}+\mathrm{BA}$ concentration in the range $510^{-6}-510^{-}$ ${ }^{4} \mathrm{M}$ (multivariate calibration) with mean errors under $10 \%$; a semiquantitative determination of the BA concentration can be deduced by difference. Finally, Ch has been successfully determined in an infant milk sample.

Keywords: Choline Oxidase; intrinsic fluorescence; Flavin Adenine Dinucleotide; Choline determination; Betaine aldehyde. 


\section{1.- Introduction}

The intrinsic spectroscopic properties of enzymes are among the most important tools for investigating the kinetic mechanisms of reactions where they take part and for obtaining information about their structural/conformational alterations. Much of this information can be derived from the UV fluorescence spectra due to several aminoacids, such as tryptophan [1,2] and, more interestingly, some additional absorbing or fluorescent groups. In this context, flavoenzymes are particularly well positioned, especially those containing FAD [3].

Free FAD can be found in two main redox forms (oxidized and reduced) and both of them in different acid/base forms. When FAD takes part of the active center of an enzyme, its chemical properties can dramatically change [3-5]. For example the hemi-reduced oxidation state (semiquinone, FAD.H), scarcely found isolated in solutions, is easily stabilized in several flavoenzymes [6,7]; in addition, the acid/base dissociation constants can also change from one enzyme to another. From the spectroscopic point of view, the molecular absorption spectra of acid/base or redox forms of flavoenzymes barely change from those of FAD in solution. However, their fluorescent properties are highly affected by the specific environment surrounding a particular enzyme. This is due not only to the quenching produced by some molecular groups near to $F A D$, but also to structural reasons. It has been demonstrated that FAD in solution can exist in two different conformations [8,9]: open (fluorescent) and stacked (non-fluorescent). Depending on the environment of a particular enzyme, one of these conformations will predominate.

During enzymatic reactions, flavoenzymes move between oxidized and reduced (or semiquinonic) forms, so their spectroscopic properties change during the reaction. Such kind of changes can be analytically used, direct or indirectly $[10,11]$, for determining the substrates involved in the corresponding reactions. However, starting from the first papers reported by Wolfbeis and Trettnak [12] this has mainly been limited to the determination of glucose using glucose oxidase [13-15]. There are several reasons for this: 1) as it has been indicated, the FAD fluorescence quantum yield cannot be predicted so it is necessary to carry out previous tests to evaluate this possibility for each particular enzyme; 2) because an excess of the substrate, compared to the enzyme, is 
generally used, the fluorescence intensity experiments some changes during the reaction and it is not easy to relate those changes in the experimental signal to the substrate concentration.

Choline oxidase $(\mathrm{ChOx})$ is an enzyme that catalyzes the oxidation of choline $(\mathrm{Ch})$ or betaine aldehyde (BA, also known as glycine betaine aldehyde) to glycine (GB, also known as glycine betaine) in bacteria. The mechanism has been studied by Gadda et al $[16,17]$ and takes place according to reaction (1). In mammals this process is catalyzed by two different enzymes: choline dehydrogenase and betaine aldehyde dehydrogenase (AIDH), respectively [18]. Reaction (1) is analytically used for choline determination, but coupled to other reactions it is also the basis of the enzymatic determination of compounds containing choline such as PAF (platelet activating factor), phosphocholine, acetylcholine, phosphatidylcholine (PC), lyso-PC or sphingomyelin, which have important roles in structural and physiological human activity. For this purpose, reaction (1) is usually coupled to a second reaction in which the hydrogen peroxide participates in a later reaction (usually enzymatic) involving a fluorophore or a chromophore [19-21]. In addition to Ch, BA is also physiologically important [22] because it protects plants from environmental stress [23] and it has several functions in mammals (for example, as a source of methyl groups) [24].

In this paper we carry out a fundamental study on the analytical possibilities of FAD fluorescence of choline oxidase (ChOx). On the one hand, several specific behaviors of the fluorescence of this enzyme have been observed; in addition, the fluorescence signals permit the quantitative determination of $\mathrm{Ch}$ and a semiquantitative estimation of $B A$ concentration in mixtures. The FAD fluorescence gives more information than that obtained when the intrinsic UV fluorescence of ChOx is used [25].

\section{2.- Experimental}

\section{1.- Apparatus}

Steady state fluorescence measurements and contour plot (3D) spectral measurements were carried out with a Photon Technology International (PTI) Time Master fluorescence spectrometer (TM-2/2003). For lifetime measurements this instrument has a $\mathrm{N}_{2}$ laser (GL-3300) that pumps a dye laser (plugged in to a frequency doubler for working with lower wavelengths) as a radiation source and a stroboscopic system as the detector. 
3D spectral measurements were carried out with a Perkin-Elmer LS 50B luminometer. $3.5 \mathrm{~mL}$ Hellma quartz cuvettes with $10 \mathrm{~mm}$ path length were used. Different bandwidths were chosen in both excitation and emission monochromators.

Absorption measurements were carried out with an Agilent 8453 UV-Visible spectrophotometer. This instrument uses a photodiode array (PDA) for simultaneous measurement of the complete UV-visible spectrum with a $1 \mathrm{~nm}$ slit.

An optical Foxy-R Oxygen Sensor (Ocean Optics), which is based on the $\mathrm{O}_{2}$ collisional quenching of fluorescence, was used for monitoring dissolved oxygen during the enzymatic reaction. Optical fibres carried both the excitation light produced by the blue LED $(450 \mathrm{~nm})$ and the fluorescence generated to the detector.

\section{2.- Reagents}

pH 8.5 to $100.1 \mathrm{M}$ carbonate solutions were prepared from solid $\mathrm{NaHCO}_{3}$ and $\mathrm{Na}_{2} \mathrm{CO}_{3}$, and pH 6 to $90.1 \mathrm{M}$ phosphate solutions from solid $\mathrm{Na}_{2} \mathrm{HPO}_{4}$ and $\mathrm{NaH}_{2} \mathrm{PO}_{4}$. Choline oxidase from Alcaligenes sp. (ChOx) and from Arthrobacter globiformis (ChOxArg) (EC 1.1.3.17) were purchased from Sigma-Aldrich C5896 as lyophilised solids with 12-15 Umg-1 activity.

Choline chloride and betaine aldehyde chloride stock solutions were supplied by Sigma (C-1879 and B3650).

\section{3.- Analytical procedures, software and data processing}

For lifetime measurements, the cuvette was filled with $\mathrm{ChOx}$ solution $\left(10 \mathrm{UmL}^{-1}\right)$ and the decay curves were obtained at pH $9\left(\lambda_{\text {exc }}=410 \mathrm{~nm} ; \lambda_{\mathrm{em}}=520 \mathrm{~nm}\right)$ and pH $6\left(\lambda_{\mathrm{exc}}=450 \mathrm{~nm} ; \lambda_{\mathrm{em}}=520 \mathrm{~nm}\right)$. To obtain the FAD lifetime, the curves were fitted to an exponential function; the best fit was determined when $0.9<\chi^{2}<1.2$.

For measurements in batch $\left(\lambda_{\mathrm{exc}}=410 \mathrm{~nm} ; \lambda_{\mathrm{em}}=520 \mathrm{~nm}\right), 1 \mathrm{~mL}$ ChOx solution and $1 \mathrm{~mL} \mathrm{pH} 9$ carbonate solution were added to the quartz cuvette $\left(\mathrm{ChO} 2 \mathrm{U} \cdot \mathrm{mL}^{-1}\right)$. After a stabilisation time of $120 \mathrm{~min}$ (this time could be suppressed or reduced but it is important to accurately measure the $F_{0}$ value), $40 \mu \mathrm{L}$ of the analyte solution (Ch o BA stock solutions or sample solution) were added to the cuvette, the solution was shaken during $5( \pm 1) \mathrm{s}$ 
(aspirating and expelling with the same micropipette used for sample addition) and the fluorescence intensity was followed over the time.

A set of 25 synthetic mixtures of Choline (Ch) and Betaine Aldehyde (BA) was prepared with 5 levels of concentration for each analyte (BA: $5.35^{*} 10^{-6} \mathrm{M}, 9.62^{*} 10^{-6} \mathrm{M}, 1.92^{*} 10^{-5} \mathrm{M}, 3.85^{*} 10^{-5} \mathrm{M}, 5.13^{*} 10^{-5} \mathrm{M}$; Ch: $5.07^{*} 10^{-}$ ${ }^{6} \mathrm{M}, 9.73^{*} 10^{-6} \mathrm{M}, 1.95^{*} 10^{-5} \mathrm{M}, 4.05^{\star} 10^{-5} \mathrm{M}, 5.67^{*} 10^{-5} \mathrm{M}$ ) (see Electronic Supplementary Material, ESM, Fig. S1) taking 18 samples for calibration and leaving out 7 for validation. The fluorescence vs. time profiles, $F=f(t)$, for each mixture was followed according to the aforementioned procedure with enzyme concentration $[\mathrm{ChOx}]=2.0$ $\mathrm{U} \mathrm{mL}-1$, at $\mathrm{pH}=9$ and $\lambda_{\mathrm{ex}}=410 \mathrm{~nm}$ and $\lambda_{\mathrm{em}}=520 \mathrm{~nm}$. Data were collected in Excel and then exported to the software UNSCRAMBLER v. 7.5 (Camo A/S, Trondheim, Norway) for normalization (every fluorescence value was divided by the initial $F$ value, $F_{0}$ ), for data pretreatment and for univariate and multivariate analysis. The chemometric procedure of calibration included a test for outliers detection based on the comparison of variances of results when the suspect sample was and was not included in the calibration set. Finally, 16 samples were used for calibration and 5 for validation (Fig. S1).

For univariate calibration, and after normalizing the experimental data, the $F_{\text {norm }}=F / F_{0}=f(t)$ profiles were obtained; then the values $\mathrm{H}_{\max }$ and $\mathrm{A}_{t}$ obtained from them (see ahead) (using the software Origin ${ }^{\mathrm{TM}}$ ) were used to build up the univariate calibration lines for $\mathrm{Ch}$ and $\mathrm{BA}$.

For multivariate calibration, and after normalization, the data were autoscaled [26]. Autoscaling supposes centering and scaling the data to avoid undesirable fluctuations during the handling of data in order to get a better interpretation of the prediction model. The leave-one-out full cross-validation procedure was used to assess the robustness of the PLS constructed models. To find the optimal number of factors or latent variables (LVs) to be used with the PLS model a previously established method was used [27]. That is, the lowest number of LVs for which the validation (cross-validation) variance value does not differ significantly from the minimum, according to an F-test with probability $P=0.25$, was chosen.

For both, the univariate and the multivariate calibrations models, and to test the prediction capability of the developed models, the statistic relative error $(R E)$ was used (2), where $d_{l}$ and $c_{i}$ are the calculated and added analyte concentrations respectively, for the mixture $i$. $R E$ can be applied to the calibration $\left(R E_{c a l}\right)$ and the 
prediction $\left(R E_{v a l}\right)$ sets. The calibration and validation (prediction) sets were defined before any data processing and remained unchanged along the work.

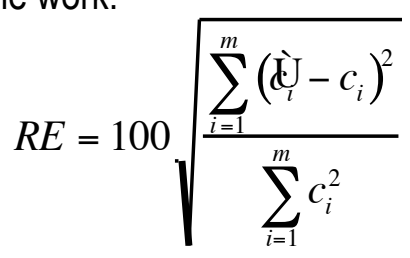

\section{4.- Milk sample treatment}

Weigh the milk powder sample (about $0.5 \mathrm{~g}$ ) in a flask, add $5 \mathrm{~mL}$ of $\mathrm{pH} 9$ carbonate buffer and shake to obtain a stable colloidal suspension ( $10 \mathrm{~min})$. The remaining supernatant is removed before going on with the treatment.

Aliquots of $1 \mathrm{~mL}$ of colloidal suspension are placed into separate Eppendorf tubes (1.5 mL capacity) and centrifuged during $30 \mathrm{~min}$ at $13400 \mathrm{rpm}$. The retrieved fluid is removed with a plastic syringe with a needle attached and filtered through a $0.20 \mu \mathrm{m}$ pore size nylon syringe filter (Albet LabScience) to remove possible traces of supernatant. The final sample fluid containing $\mathrm{Ch}$ is then collected and the univariate procedure is applied.

\section{3.- RESULTS AND DISCUSSION}

\section{1.- Excitation and fluorescence spectra.}

As it occurs with most enzymatic reactions, acidity affects the kinetics of the Choline (Ch) or the BetaineAldehyde (BA) enzymatic oxidations catalysed by choline oxidase; as will be shown later, for $\mathrm{pH} \leq 6$ and $\mathrm{pH}>10$ the reaction is not observed, but here we are interested in showing the spectroscopic behaviour of $\mathrm{ChOx}$. In this $\mathrm{pH}$ range, the molecular absorption spectrum of choline oxidase from Alcaligenes sp (ChOx) remains almost unchanged (Fig. S2) and presents the expected maxima due to FAD at 350 and $450 \mathrm{~nm}$. However, a different behaviour is observed when fluorescence is being measured. Fig. 1 compares the $3 \mathrm{D}$ spectra of ChOx at two $\mathrm{pH}$ values, 6 and 9 . In both cases the fluorescence maximum appears at $520 \mathrm{~nm}$, but the excitation maximum at $\mathrm{pH}=9$ has suffered a hypsochromic displacement of about $50 \mathrm{~nm}$ compared to the absorption spectra; this is not observed at $\mathrm{pH}=6$. Similar studies were carried out using choline oxidase from Arthrobacter globiformis 
$\left(\mathrm{ChOX}_{\mathrm{Arg}}\right)$ with similar results. As far as we know, this fact has not been previously reported for flavoenzymes or for fluorophores in general. In order to clarify this behaviour, the following additional results should be taken into account:

1) The fluorescence lifetime values obtained for $\mathrm{ChOx}$ at $\mathrm{pH}=6$ and 9 were not significantly different $(4.8 \pm 0.2 \mathrm{~ns}$ and $5.0 \pm 0.2 \mathrm{~ns}$, respectively), so similar deactivation processes from the excited state seem to occur in both cases. Because of that, the displacement of the excitation maximum does not go in parallel with proton or electron transfer in the excited state, i.e. there is not a photoinduced chemical process; in fact, this effect was observed when working at different powers of the radiation source. In addition and according to the bibliography [3], the only FAD species which is able to give the absorption spectra shown in Fig. $1 \mathrm{~A}$ is the protonated form of FAD $\left(F A D \cdot H^{+}\right)$, but, this species is not fluorescent.

2) During the enzymatic reaction $F A D$ is reduced to $F A D \cdot H_{2}$. According to Fig. 1, the excitation maximum of the reduced form working at $\mathrm{pH}=9$ (at $\mathrm{pH}=6$ there is no reaction) does not differ from those obtained by molecular absorption spectrometry (ESM, Fig. S2), which indicates that the displacement only affects the oxidized form of the enzyme.

3) According to the bibliography $[28,29]$, the spectrum of the isoalloxazine structure contained in FAD is prone to be modified by polarity changes.

Taking all these arguments into account it can be concluded that the photoinduced electron or proton transfer processes in the excited state (after absorption) do not seem to be responsible for the displacement observed; more probably, a polarity change in the $\mathrm{ChOx}$ molecule could be charged with that responsibility and the effect would entail the capability to react with the substrate..

\section{2.- Mechanism of the reaction: Analytical signals.}

Figures 2 and 3 show the fluorescence intensity variation of $\mathrm{ChOx}$ during the enzymatic reaction when $\mathrm{BA}$ or $\mathrm{Ch}$ are used as substrates in the given experimental conditions, together with excitation spectra at representative reaction times. After the addition of BA (Fig. 2), the initial fluorescence intensity $\left(F_{0}\right)$ shows an instantaneous decrease $\left(F_{\text {min }}\right)$ due to the $F A D$ reduction to $F A D . H_{2}$, which is maintained until all $B A$ is oxidized to glycine 
betaine $(G B)$. Then FAD is regenerated and the $F_{0}$ value is obtained again (because of the dilution, when the analyte volume added is high enough the final $F$ value does not match $\left.F_{0}\right)$; the time along which the enzyme shows a lower fluorescence corresponds to a steady-state. In the concentration range tested, the $F_{\min }$ value is scarcely dependent of the BA concentration, but several other parameters of the profile (ESM, Fig. S3), do depend on it; finally, the total area $\left(A_{T}\right)$, as defined in Fig. 2A, was used. In order to avoid the uncertainty due to the $\mathrm{ChOx}$ concentration, the $\mathrm{F}_{\text {norm }}$ parameter (obtained by the quotient of $\mathrm{F}$ divided by $\mathrm{F}_{0}$ ) has frequently been used throughout the paper.

When the reaction is carried out with $\mathrm{Ch}$ (Fig. 3A), the representation resembles that obtained for BA but an additional fluorescence intensity maximum $\left(F_{\max }\right)$ at the beginning of the reaction is observed before the intensity drooping to $F_{\min }$. This result can be explained considering that the second part of the profile mainly corresponds to the oxidation of the $B A$ to $G B$, whereas the first part of the profile mainly corresponds to the oxidation of $\mathrm{Ch}$ to $\mathrm{BA}$. In addition to $\mathrm{A}_{T}, \mathrm{H}_{\max }$ defined as the difference between the maximum and the minimum intensities $\left(\mathrm{H}_{\max }=\mathrm{F}_{\max }-\mathrm{F}_{\min }\right)$, is also related with the Ch concentration (ESM, Fig. S4). From a kinetic point of view, these results show that the reaction could follow two consecutive steps: in the first one the Ch present in the solution is oxidized to $B A$ and in the second one $B A$ is oxidized to $G B$; however overlapping of both steps is also compatible with the obtained results (see below). Similar profiles were observed using ChOxArg. Again, when the reaction is measured by molecular absorption spectrometry, no differences were obtained regarding the absorbance profile during the reaction from $\mathrm{Ch}$ to $\mathrm{BA}$.

From the analytical point of view, these results open new possibilities to the determination of both analytes, but it also permits us to obtain some information about the mechanism of the reaction. This would have not been possible should the enzymatic reaction had been followed through the conventional procedure based on $\mathrm{O}_{2}$ monitorization, neither when coupling an enzymatic reaction for $\mathrm{H}_{2} \mathrm{O}_{2}$, nor when the reaction is followed by UVvis molecular absorption spectrometry. Moreover, when the enzymatic reaction is followed at the tryptophan wavelengths [25], the changes in the fluorescence intensity between both processes are not so evident, so it is difficult to differentiate each of the single steps (ESM, Fig. S5). 
In order to obtain more information about the origin of this signal (and possibly about the mechanism of the reaction), the changes in the $\mathrm{ChOx}$ spectra during the enzymatic reaction with $\mathrm{Ch}$ or $\mathrm{BA}$ were monitored in detail. The most interesting differences appear in the excitation spectra. During the enzymatic reaction with BA (Fig. 2B), and just after the addition of the analyte, the wavelengths of the maximum moves to about $360 \mathrm{~nm}$, as expected for the FAD. $\mathrm{H}_{2}$ formation, but no additional changes in the wavelengths were observed during the reaction. Only modifications in the intensity (due to $\mathrm{FAD} . \mathrm{H}_{2}$ formation) are appreciable. When the analyte was Ch (Fig. 3B), during the initial stages of the reaction (corresponding to the $\mathrm{H}_{\max }$ appearance), the spectra showed a significant displacement of about $30 \mathrm{~nm}$; after these initial stages (i.e., when the fluorescence intensity decreased to the $F_{\text {min }}$ ) the spectra moved to the FAD. $\mathrm{H}_{2}$ wavelength obtained with $B A$. The displacement in the excitation spectra was correlated with slight changes in the fluorescence spectra (ESM, Fig. S6). Similar results were observed using $\mathrm{ChOx}_{\text {Arg. }}$.

These differences could be explained in terms of changes in the environment surrounding FAD; this could also be an argument to explain the difference between the excitation and molecular absorption spectra. However, a more probable hypothesis comes from the fact that the reduced form of the Flavin during the first part of the oxidation of $\mathrm{Ch}$ is different from that of the corresponding process with BA. The semiquinonic species (FAD. $\left.\mathrm{H}^{-}\right)$ could be the intermediate. When the reaction is followed through the $\mathrm{O}_{2}$ consumed during the reaction (ESM, Figs. S7 and S8), it seems clear that the kinetic mechanisms for both analytes are different: BA seems to follow a logarithmic curve, but $\mathrm{Ch}$ seems to fit a sigmoidal curve. In any case, the amount of $\mathrm{O}_{2}$ consumed by $\mathrm{Ch}$ to produce $B A$ is approximately the amount consumed by $B A$, to produce $G B$, approaching the stoichiometry of the reaction (1).

\section{3.- Optimization of the experimental conditions. Analytical signals}

\section{A) ChOx concentration}

The most important variables affecting the analytical applications of the reaction are $\mathrm{pH}$, enzyme concentration and the nature of the enzyme. The enzyme kinetics dictates that the higher the enzyme concentration, the faster the reaction. In enzymatic methods of determination, based on colorimetric detection of $\mathrm{H}_{2} \mathrm{O}_{2}$ or on $\mathrm{O}_{2}$ 
consumption, a higher enzyme concentration may be preferred in order to obtain a higher sensitivity and/or a faster method. However, this is not the case when the signal of the enzyme is being measured (ESM, Fig. S9). Low concentrations of the enzyme provide longer reaction times and thus higher values of the analytical signal and higher sensitivity (table 1); so, the enzyme concentration must be fitted to the requirements of the application.

Another relevant analytical conclusion is reached when a too high enzyme concentrations is used. The increase in the reaction rate makes impossible to detect the value of $F_{\max }$ at the beginning of the reaction, thus $F_{\max }$ does not appear and it is not possible to differentiate Ch from BA. In these cases, both analytes seem to obey the same reaction pattern.

\section{B) The effect of the $\mathrm{pH}$.}

As it has been indicated above, the $\mathrm{pH}$ affects the spectrum of $\mathrm{ChOx}$, especially that of excitation. As Fig. 4A shows, at $\mathrm{pH}=8-10$ the maximum of the excitation spectra does not agree with the maxima obtained in the absorption spectra (ESM, Fig. S2), with a higher intensity at $\mathrm{pH}=9$ (which again discards an acid/base process in the excited state). However, at $\mathrm{pH}=6$ and 7 , the maximum at $400 \mathrm{~nm}$ splits into two maxima, whose wavelengths match the absorption spectra.

In order to show how these changes affect the reaction kinetics and the sensitivity of the analytical method, Fig. 4B shows the $F_{\text {norm }}=f(t)$ profiles obtained working at $400 \mathrm{~nm}$ excitation (in these experiments the enzyme concentration was maintained high in order to see the differences more clearly). As it can be seen, in the range from $\mathrm{pH}=8-10$, the shape of the signals agree with the results previously shown; at $\mathrm{pH}=10$ the reaction is slower than at $\mathrm{pH} 8$ and 9 (similar in both cases) and at $\mathrm{pH}=9$ the signal is higher because of the absolute value of the fluorescence intensity. At $\mathrm{pH}=6$, there is no reaction. At $\mathrm{pH}=7$, since the maximum does not appear at $400 \mathrm{~nm}$ (Fig. 4A), the fluorescence intensity after the Ch addition increases up to $F_{\max }$, as expected, and then it decreases in a similar manner to the rest of $\mathrm{pH}$ values. However, when the excitation spectra are taken during the reaction, it is concluded that the final spectra of the $\mathrm{ChOx}$ do not match either with the initial spectra or with absorption spectra (ESM, Fig. S3). This means that the final state of the enzyme is not the oxidized form (as it 
occurs at other $\mathrm{pHs}$ ) but another form, and, in consequence, the reaction mechanism at this $\mathrm{pH}$ does not seem to fit with that observed at $\mathrm{pH}=8-10$.

Finally, it is important to indicate that both types of enzymes have a similar behaviour. Because of ChOX Arg generates smaller fluorescence changes between the two species (FAD and FAD.H $)$ (ESM, Figs. S10 and S11), the preparation from Alcaligenes sp was always used.

The detailed studies performed by Gadda et al $[16,17]$ about the kinetics and the mechanism of the ChOx enzymatic reaction are a very important bibliographic reference. From these studies, it can be deduced that the Ch oxidation is sequential, i.e. when $\mathrm{ChOx}$ links to a $\mathrm{Ch}$ molecule, both oxidation processes (first up to BA and later on up to GB) are sequentially produced. They found this conclusion after working mainly at $\mathrm{pH}=7$. As it can be deduced from the results above, the mechanism of the reaction may change depending on the experimental conditions, so both the mechanism proposed by Gadda and that shown in this paper are likely to occur depending on the experimental conditions used.

\section{C) Analytical signals}

In the optimal conditions, analytical signals such as those in Fig. $2 \mathrm{~A}$ and $3 \mathrm{~A}$ were obtained. $\mathrm{H}_{\max }$ can be related to Ch concentration; on the other hand, the value of $A_{T}$ can be related to BA concentration regardless it comes from a species initially present in the sample or it has been generated from the original Ch (reaction (1)); so the value of $A_{T}$ should be related to the initial concentration of $C h+B A$ or to any of them whenever only one is initially present. The individual relations are compiled in Table 2, they were obtained using pure solutions of either Ch or BA and they could theoretically be used as calibration lines for both analytes. Note that the slope of the $A_{T}$ calibration line obtained for both, $C h$ and $B A$ are similar which indicates that $A_{T}$ is mainly due to the $B A$ $\rightarrow$ GB step. When using $A_{T}$ as analytical signal the response range could be increased by using a second order calibration line. In both cases the RSD value was under $5 \%$.

The linear response range can be empirically enlarged by using $1 / A_{T}$ as the analytical signal, but precision gets smaller and the statistical problem to manage errors increases. 


\section{4.- $\mathrm{Ch}$ and BA selective and joint determinations.}

\section{A) Univariate calibration}

The results obtained above show that the intrinsic fluorescence of the flavin in ChOx can be used for either the single determination of $\mathrm{Ch}$ (using $\mathrm{H}_{\max }$ or $\mathrm{A}_{T}$ ) or $\mathrm{BA}$ (using $\mathrm{A}_{\mathrm{T}}$ ) in samples containing only one of both analytes. Taking into account that the $\mathrm{H}_{\max }$ value is only due to $\mathrm{Ch}$, it is reasonable to use that signal for the selective determination of $\mathrm{Ch}$ in the presence of $\mathrm{BA}$. In addition, by the use of the $\mathrm{A}_{T}$ value the joint determination of Ch+BA can also be set out; finally, after combining the previous results, the concentration of BA can be deduced.

The experimental section describes the calibration-validation matrix (ESM, Fig. S1) prepared from mixtures having known amounts of $\mathrm{Ch}$ and $\mathrm{BA}$. The $\mathrm{F}_{\text {norm }}=\mathrm{f}(\mathrm{t})$ profiles obtained for those reaction mixtures are given in Fig. S12. As an example, Fig. 5 shows two series of reaction profiles in which the effect of $\mathrm{Ch}$ and $\mathrm{BA}$ on the experimental signal can be seen. Both analytes contribute to the experimental signal, so each of them interfere the signal of the other when measuring $A_{T}$; on the other hand, BA unexpectedly affects the $H_{\max }$ value and this requires some extra explanation.

At the beginning of the reaction $\mathrm{ChOx}$ reacts with both $\mathrm{BA}$ and $\mathrm{Ch}$; so there is a competence of both substrates to link with the enzyme. By comparing figures $2 \mathrm{~A}$ and $3 \mathrm{~A}$ it can be deduced that the time required for the oxidation of $\mathrm{Ch}$ to $\mathrm{BA}$ is much lower than that necessary for the oxidation of $\mathrm{BA}$ to $\mathrm{GB}$. This means that the reaction of Ch with the enzyme is much faster than with BA (Figs. 2 and 3) and because ChOx is in defect, the most of the enzyme can be supposed to be linked with $\mathrm{Ch}$; however, the higher the BA concentration, the lower the amount of $\mathrm{ChOx}$ available for $\mathrm{Ch}$ and, consequently, the value of $\mathrm{F}_{\max }$ will increase. On the other hand, the enzyme is reacting with $B A$, and the initial fluorescence (and in consequence, $F_{\max }$ ) value tends to decrease (Fig. 2); so the higher the BA concentration, the lower the initial fluorescence. The former effect is probably more important than the latter but, as a whole, both effects tend to balance and the final interference of BA in $H_{\max }$ results minimized.

Table 3 gives the calibration line for [Ch] using $\mathrm{H}_{\max }$ as analytical signal from the calibration mixtures of the Calibration-Validation Matrix (CVM); that is, the interference of BA is included in the calibration line. The straight 
line equation matches reasonably well the one obtained with pure Ch solutions (Table 2), indicating that BA does not severely interfere the $\mathrm{Ch}$ determination. The RE values for the calibration and validation sets were about $10 \%$ in both cases. This method presents very few interferences, because molecules structurally similar to choline (for example, ethanol amines) barely interfere [30]; in classical methodologies involving ChOx, interferences come from the later colorimetric or amperometric determination of $\mathrm{H}_{2} \mathrm{O}_{2}$ generated in the $\mathrm{ChOx}$ [31]; our method is virtually free of this type of interferences.

The determination of $[\mathrm{Ch}]+[\mathrm{BA}]$ using the $\mathrm{A}_{T}$ values as analytical signal was carried out in a similar way. Table 3 provides the calibration line, and the RE values are also given. Taking into account the higher errors obtained, the method, in this case, can only be considered semiquantitative. From these results it is also possible to obtain the $[\mathrm{BA}]$ concentration by difference. Despite of the random errors of both results are not extremely high, the propagation of uncertainty through the squares effect generated unbearable mean errors in [BA] higher than $40 \%$ (table 3 ) indicating that the BA concentration should not be estimated by this method.

\section{B) Multivariate calibration}

Partial Least Squares (PLS) has been used as the calibration algorithm, trying to make the most of the fluorescence-time profiles and different models have been tested based on the selection of different variables (time intervals) and the number of latent variables (LVs); in any case, $t=0$ corresponds to the moment of the substrate addition. According to the basis of the method one should expect that the first part of the profile could be suitable for Ch determination while the final part of the profile (longer times) could better represent the joint concentration of both analytes. The variable selection for PLS was applied using the Martens' uncertainty test [32]. This test, available in The Unscrambler@ application) uses a jackknifing procedure to identify nonsignificant variables. According to this procedure the time intervals selected for Ch determination were 3-15, 3345 and 113-121s.

The results are also compiled in Table 3. As can be seen, multivariate calibration provides lower errors than univariate calibration for both $\mathrm{Ch}$ and $\mathrm{Ch}+\mathrm{BA}$ determinations; however, calibration and validation errors are unbalanced and some tendency to overfitting can be observed. 
In this case the results for BA determination (as a difference between $\mathrm{Ch}+\mathrm{BA}$ less $\mathrm{Ch}$ ) are much lower than those obtained through univariate calibration and the method could be recommended for the semiquantiative determination of $\mathrm{BA}$ in samples containing $\mathrm{BA}$ and $\mathrm{Ch}$.

Determination $\mathrm{Ch}$ and $\mathrm{BA}$ are not very usual but, as has been indicated above, it is important in biological choline metabolism studies and in the metabolism of different plants

\section{4.- Ch determination in a milk sample.}

Finally, the univariate method was applied to Ch determination in a milk infant formula; in this kind of samples BA is not present. The procedure described in the Experimental section (univariate calibration) was applied. The sample solution showed an intense fluorescence in the UV zone (probably due to proteins) and a less intense signal at the characteristic wavelengths of $\mathrm{ChOx}\left(\lambda_{\mathrm{exc}}=450 \mathrm{~nm}\right.$ and $\left.\lambda_{\mathrm{em}}=520 \mathrm{~nm}\right)$. This signal is probably due to riboflavin (which is present in milk as Vitamin B2,) and produced a background which upset the $\mathrm{ChOx}$ fluorescence; additional studies shown that working at $\mathrm{pH}=9$ (carbonate buffer) the background could be minimized.

In these conditions, Ch was determined (in triplicate) by interpolation in a calibration line obtained in a similar way with concentrations ranging between $7.5010^{-6}$ and $4.7510^{-5} \mathrm{M}$. A concentration of $0,386( \pm 0.020) \mathrm{mg} \mathrm{g}^{-1}$ Ch was obtained. Using the same solution, Ch was also determined (in triplicate) by a colorimetric enzymatic method based on ChOx, Horseradish peroxidase (HRP) and ABTS; the reference method gave 0,400 $( \pm 0.015)$ $m g \mathrm{~g}^{-1}$. The obtained results do not differ significantly $(P=0,05)$.

\section{5.- Conclusions}

From the fundamental studies shown in this paper it can be deduced that for those enzymes in which FAD fluorescence can be observed (i.e., the open structure predominates over the stacked form), valuable information about the mechanism of the enzyme-catalyzed reaction can be obtained from the fluorescence excitation spectra. This paper shows that the intrinsic fluorescence of the enzyme can also be useful from an analytical point of view. It may permit substrates to be determined (Ch in this case) in a reagentless mode (no 
fluorophores as indicators need to be used), setting out the basis for this kind of sensor. On the other hand, in some particular cases, singular applications, such as the detection of reaction intermediates (in this case BA) or the determination of the joint $[\mathrm{Ch}]+[\mathrm{BA}]$ concentration can be obtained.

\section{6.- Acknowledgements}

The authors thank the MINECO of Spain (project CTQ2012-34774), the Gobierno de Aragon (DGA-FEDER) (Research Group E-74) and UPV/EHU (project GIU13/15) for founding support.

\section{7.- References}

1. R. Lakowicz, Topics in Fluorescence Spectroscopy, Volume 6 :Protein Fluorescence, Kluwer Academic Press, New York, 2002.

2. N. Vekshin N, Photonics of biopolymers (Biological and Medicinal Physics Series), Springer-Verlag, Berlin, 2002.

3. E. Silva, A.M. Edwards AM, Flavins: Photochemistry and Photobiology. In: D.P. Häder and G. Jori (series Eds.) Comprehensive series in Photochemical and Photobiological Sciences Vol. 6. RSC Publishing, European Society for Photobiology, 2006.

4. Y.T. Kao, C. Saxena, T. He, L. Guo, L. Wang, A. Sancar, D. Zhong, Ultrafast Dynamics of flavins in five redox states, J. Am. Chem. Soc. 130 (2008) 13132-13139.

5. Y.T. Kao, C. Sang, S. Song, N. Ozturk, L. Li, L. Wang, A. Sancar, D. Zhong , Ultrafast Dynamics and Anionic Active Sites of the Flavin Cofactor in Cryptochrome and Photolyase, J. Am. Chem. Soc. 130 (2008) 7695-7701.

6. V. Massey, G. Palmer, On the existence of spectrally distinct classes of flavoprotein semiquinones. A new method for the quantitative production of flavoprotein semiquinones, Biochemistry 5 (1966) 31812189. 
7. G. Gadda, Hydride transfer made easy in the reaction of alcohol oxidation catalyzed by flavin-dependent oxidases, Biochemistry 47 (2008) 13745-13753.

8. S.D.M. Islam, T. Susdorf, A. Penkofer, P. Hegemann, Fluorescence quenching of flavin adenine dinucleotide in aqueous solution by $\mathrm{pH}$ dependent isomerisation and photo-induced electron transfer, Chem. Phys. 295 (2003) 137-149.

9. J. Brazard, A. Usman, F. Lacombat, C. Ley, M.M. Martin, P. Plaza, New insights into the ultrafast photophysics of oxidized and reduced FAD in solution, J. Phys. Chem. A 115 (2011) 3251-3262.

10. J. Galban, Y. Andreu, J.F. Sierra, S. de Marcos, J.R. Castillo, Intrinsic fluorescence of enzymes and fluorescence of chemically modified enzymes for analytical purposes: a review, Luminiscence 16 (2001) 199-210.

11. J. Galban, I. Sanz-Vicente, E. Ortega, M. del Barrio, S. de Marcos, Reagentless fluorescent biosensors based on proteins for continuous monitoring systems, Anal. Bioanal. Chem. 402 (2012) 3039-3054.

12. W. Trettnak, O.S. Wolfbeis, Fully reversible glucose biosensor based on the intrinsic fluorescence of glucose oxidase, Anal. Chim. Acta 221 (1989) 195-203.

13. R. Esposito, I. Delfino, M. Lepore, Time-resolved flavin adenine dinucleotide fluorescence study of the interaction between immobilized glucose oxidase and glucose, J. Fluoresc. 23 (2013) 947-955.

14. M. Portaccio, M. Lepore, B. Della Ventura, O. Stoilova, N. Manolova, I. Rashkov, D.G. Mita, Fiber-optic glucose biosensor based on glucose oxidase immobilised in a silica gel matrix, J. Sol-Gel. Sci. Technol. 50 (2009) 437-448.

15. I. Chudobova, E. Vrbova, M. Kodicek, J. Janovcova, J. Kas, Fiber optic biosensor for the determination of D-glucose based on absorption changes of immobilized glucose oxidase, Anal. Chim. Acta 319 (1996) 103-110.

16. G. Gadda, Kinetic mechanism of choline oxidase from Arthrobacter globiformis, Biochim. Biophys. Acta 1646 (2003) 112-118.

17. F. Fan, G. Gadda G, On the catalytic mechanism of choline oxidase, J. Am. Chem. Soc. 127 (2005) 20672074. 
18. S.A.S. Craig, Betaine in human nutrition, Am. J. Clin. Nutr. 80 (2004) 539-549,

19. H. Hidaka, K. Yamauchi, H. Ohta, T. Akamatsu, T. Honda, T. Katsuyama, Specific, rapid and sensitive enzymatic measurement of sphingomyelin, phosphatidylcholine and lyso-phosphatidylcholine in serum and lipid extracts, Clin. Biochem. 41 (2008) 1211-1217.

20. M.R. Hojjati, X.C. Jiang, Rapid, specific, and sensitive measurement of plasma sphingomyelin and phosphatidylcholine, J. Lip. Res. 47 (2006) 673-676.

21. M.D. Marazuela, M.C. Moreno-Bondi, Determination of choline containing phospholipids in serum with a fiber optic biosensor, Anal. Chim. Acta 347 (1998) 19-29.

22. H. Allouche-Arnon, A. Gamliel, J. Sosna, J.M. Gomori, R. Katz-Brul, In vitro visualization of betaine aldehyde synthesis and oxidation using hyperpolarized magnetic resonance spectroscopy, Chem. Comm. 49 (2013) 7076-7078.

23. M.K. Chern, D.A. Cage, R. Pietruszko, Betaine aldehyde, betaine and choline levels in rat livers during ethanol metabolism, Biochem. Pharm. 60 (2000) 1629-1637.

24. C. Lerma, P.J. Rich, G.C. Ju, W. Yang, A.D. Hanson, D. Rhodes, Betaine Deficiency in Maize. 1 Complementation Tests and Metabolic Basis, Plant. Physiol. 95 (1991) 1113-1119.

25. J. Galbán, O. Sánchez-Monreal, Y. Andreu, S. de Marcos, J.R. Castillo, Choline determination based on the intrinsic and the extrinsic fluorescence of choline oxidase, Anal. Biochem. 334 (2004) 207-215.

26. R. Kramer, Chemometrics Techniques for Quantitative Analysis. Marcel Dekker, New York, 1998.

27. D.M. Haaland, E.V. Thomas, Partial least-squares methods for spectral analyses. 1. Relation to other quantitative calibration methods and the extraction of qualitative information, Anal, Chem. 60 (1988) 1193-1202.

28. P. Drossler, W. Holzer, A. Penzkofer, P. Hegemann, pH dependence of the absorption and emission behavior of riboflavin in aqueous solution, Chem. Phys. 282 (2001) 429-439.

29. M. Wu, L.A. Eriksson, Absorption Spectra of Riboflavins. A Difficult Case for Computational Chemistry, J Phys Chem A 114 (2010) 10234-10242. 
30. M.A. Roulier, B. Palenik, F.M.N. Morel, A method for the measurement of choline and hydrogen peroxide in water, Mar. Chem. 30 (1990) 409-421.

31. A. Yumura, E. Hokazono, K. Ikeda, K. Obayashi, Y. Ando, S. Osawa, Development of the enzymatic assay for the whole blood choline using an automated biochemical analyzer, Int. J. Anal. Bio-Sci 2 (2014) 23-32.

32. K.H. Esbensen, Multivariate Data Analysis in Practice, $5^{\text {th }}$ ed., CAMO Software AS, Norway, 2009. 


\section{Figure Captions}

Fig. $13 \mathrm{D}$ fluorescence contour plot obtained for $\mathrm{ChOx}\left(10 \mathrm{U} \mathrm{mL}^{-1}\right)$ at $\mathrm{pH}=6(\mathbf{A})$ and 9 (B) before the enzymatic reaction. During the enzymatic reaction with $\mathrm{Ch}\left(2.2^{*} 10^{-3} \mathrm{M}\right)$, the spectrum given in $\mathrm{C}$ was obtained at both $\mathrm{pH}$. $\mathrm{X}$-axis is the excitation wavelength $\left(\lambda_{\text {exc }}\right), Y$-axis the fluorescence wavelength $\left(\lambda_{\text {em }}\right)$ and the show-card to the right shows fluorescence intensity (arbitrary units)

Fig. 2 Modification of the ChOx fluorescence during the reaction with $B A\left([B A]=1.65^{\star} 10^{-4} \mathrm{M}, \mathrm{pH}=9\right.$, $[\mathrm{ChOx}]=1.8$ $\mathrm{U} \mathrm{mL}^{-1}, \lambda_{\mathrm{exc}}=410 \mathrm{~nm}$ and $\left.\lambda_{\mathrm{em}}=520 \mathrm{~nm}\right)$

A) Variation of the fluorescence intensity profile

B) Variation of the excitation fluorescence spectra during the enzymatic reaction: (0) before BA addition, (1) $4 \mathrm{~s}$ after the beginning of the reaction, (2) $50 \mathrm{~s}$ after the beginning of the reaction

Fig. 3 Modification of the ChOx fluorescence during the reaction with $\mathrm{Ch}\left([\mathrm{Ch}]=1.45^{\star} 10^{-4} \mathrm{M}, \mathrm{pH}=9,[\mathrm{ChOx}]=1.8\right.$ $\mathrm{U} \mathrm{mL}^{-1}, \lambda_{\mathrm{exc}}=410 \mathrm{~nm}$ and $\left.\lambda_{\mathrm{em}}=520 \mathrm{~nm}\right)$

A) Variation of the fluorescence intensity profile

B) Variation of the excitation fluorescence spectra during the enzymatic reaction: (0) before Ch addition, (1) $4 \mathrm{~s}$ after the beginning of the reaction, (2) $50 \mathrm{~s}$ after the beginning of the reaction

Fig. $4 \mathrm{pH}$ effect on the fluorescence excitation spectra and profiles ( $[\mathrm{ChOx}]=2.0 \mathrm{U} \mathrm{mL}-1,1.2^{*} 10^{-4} \mathrm{M} \mathrm{Ch}$ )
A) Fluorescence excitation spectra $\lambda_{\mathrm{em}}=520 \mathrm{~nm}$ ).
(a) $\mathrm{pH}=10$,
(b) $\mathrm{pH}=9$,
(c) $\mathrm{pH}=8$,
(d) $\mathrm{pH}=7$,
(e) $\mathrm{pH}=6$
B) $F_{\text {norm }}=f(t)\left(\lambda_{\text {exc }}=410 \mathrm{~nm}\right.$ and $\left.\lambda_{\mathrm{em}}=520 \mathrm{~nm}\right)$.
(a) $\mathrm{pH}=10$,
(b) $\mathrm{pH}=9$,
(c) $\mathrm{pH}=8$,
(d) $\mathrm{pH}=7$,
(e) $\mathrm{pH}=6$

Fig. $5 \mathrm{~F}=\mathrm{f}(\mathrm{t})$ profiles obtained for several synthetic samples $\quad\left(\mathrm{pH}=9,[\mathrm{ChOx}]=2 \mathrm{U} \mathrm{mL}^{-1}, \lambda_{\mathrm{exc}}=410 \mathrm{~nm}\right.$ and $\lambda_{\mathrm{em}}$ $=520 \mathrm{~nm})$
A) $1.92 * 10-5 \mathrm{M}$ BA concentration
(a) $5.07^{*} 10^{-6} \mathrm{M} \mathrm{Ch},(b)$
(b) $9.70^{*} 10^{-6} \mathrm{M} \mathrm{Ch}$
, (c) $1.95^{*} 10^{-5} \mathrm{M} \mathrm{Ch}$
(d) $4.05^{*} 10^{-5} \mathrm{M} \mathrm{Ch}$,
(e) $5.67^{*} 10^{-5} \mathrm{M} \mathrm{Ch}$
B) $9.7^{*} 10^{-6} \mathrm{M}$ Ch concentration.
(a) $5.35^{\star} 10^{-6} \mathrm{M} \mathrm{BA}$,
(b) $9.6^{*} 10^{-6} \mathrm{M} \mathrm{BA}$,
(c) $1.92^{*} 10^{-5} \mathrm{M} \mathrm{BA}$, (d) $3.85^{*} 10^{-5} \mathrm{M} \mathrm{BA}$,
(e) $5.13^{*} 10^{-5} \mathrm{M} \mathrm{BA}$ 
Fig 1

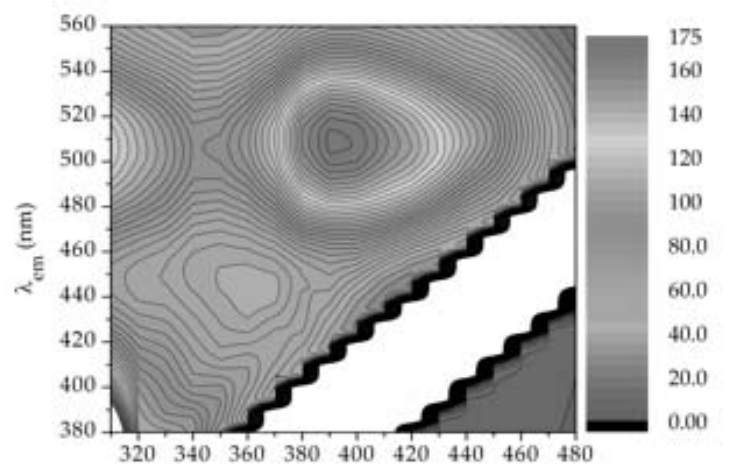

A

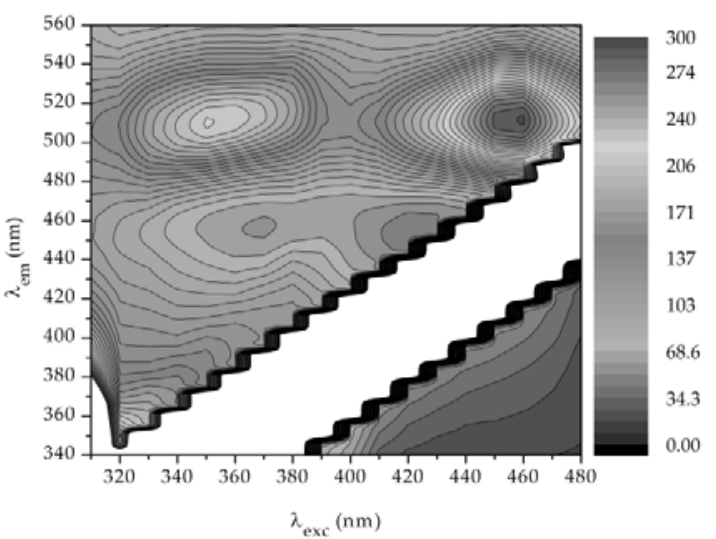

B

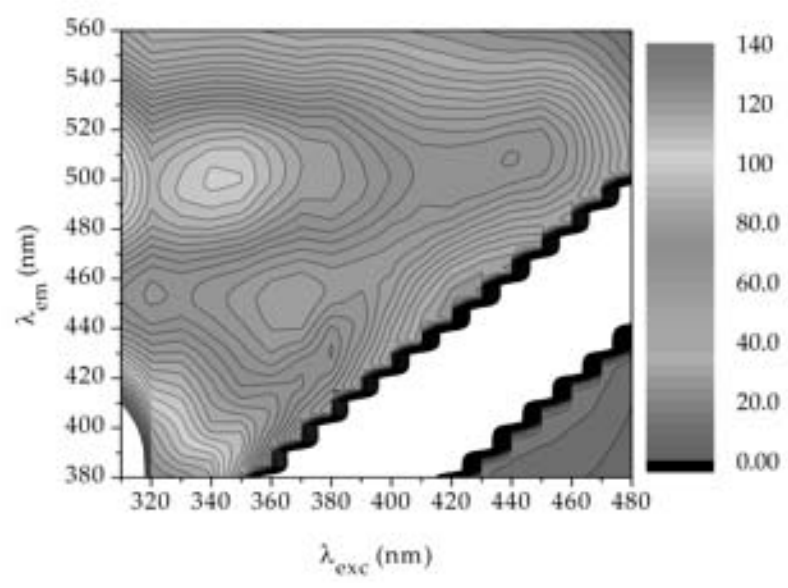

C 
Fig 2

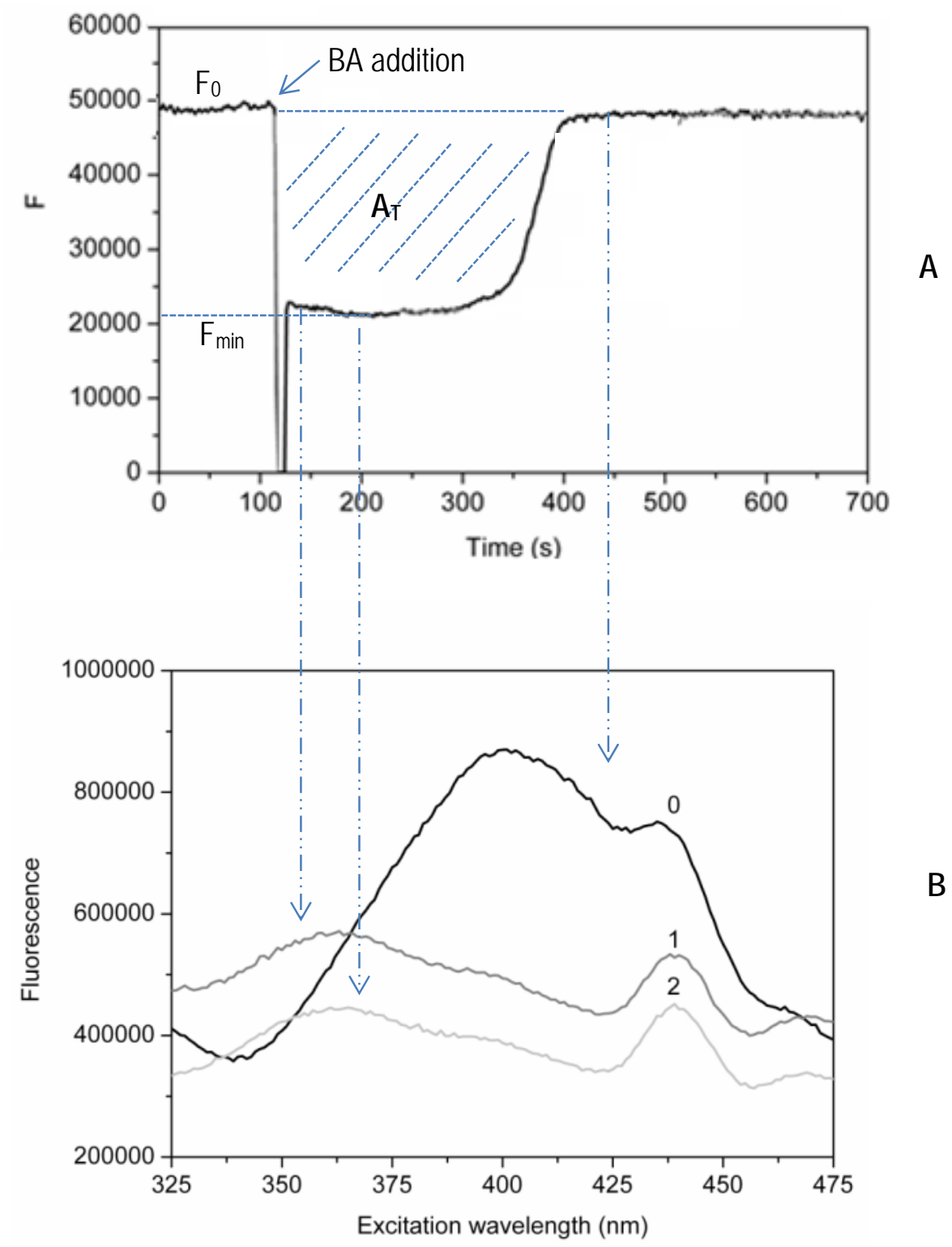


Fig 3

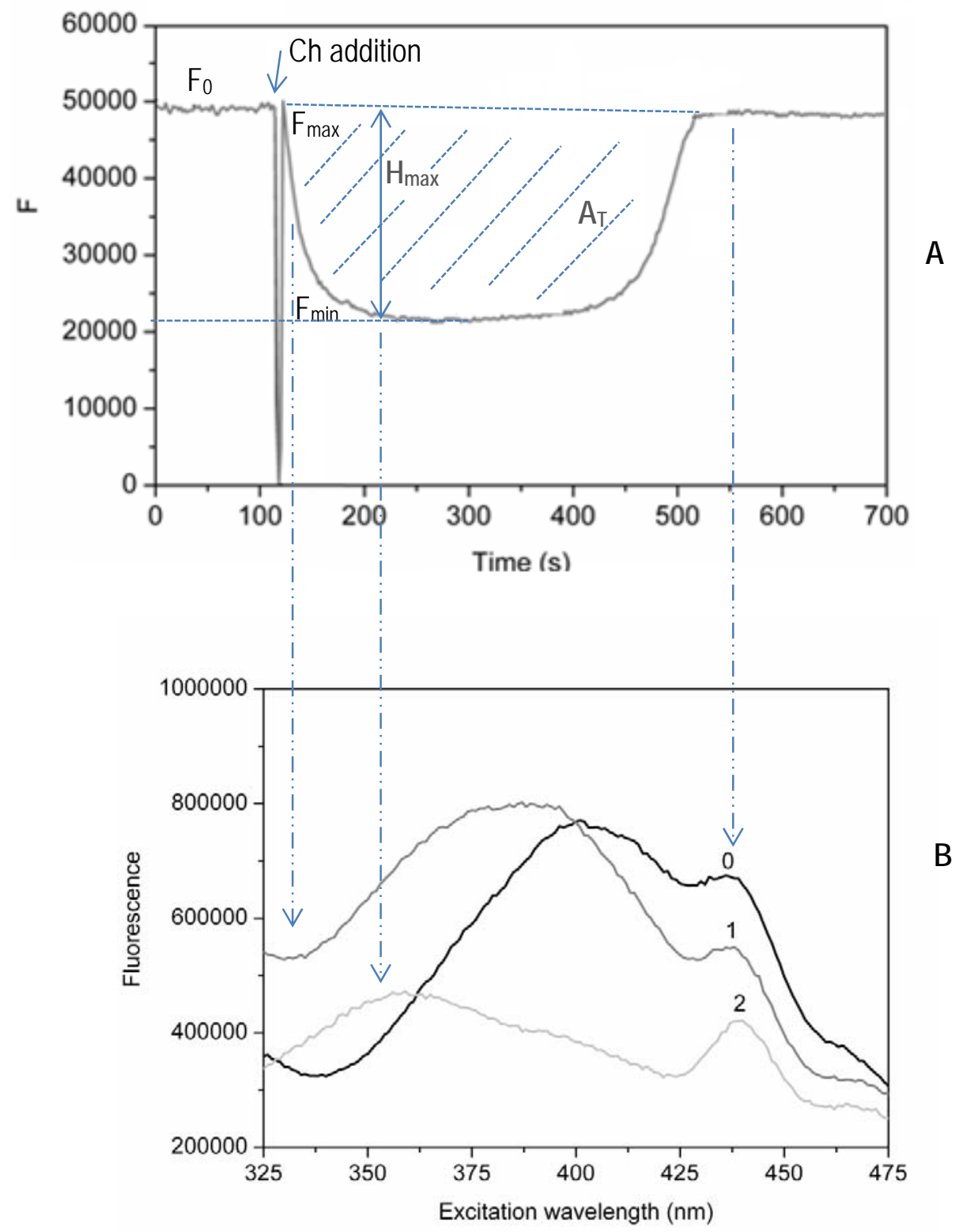


Fig 4

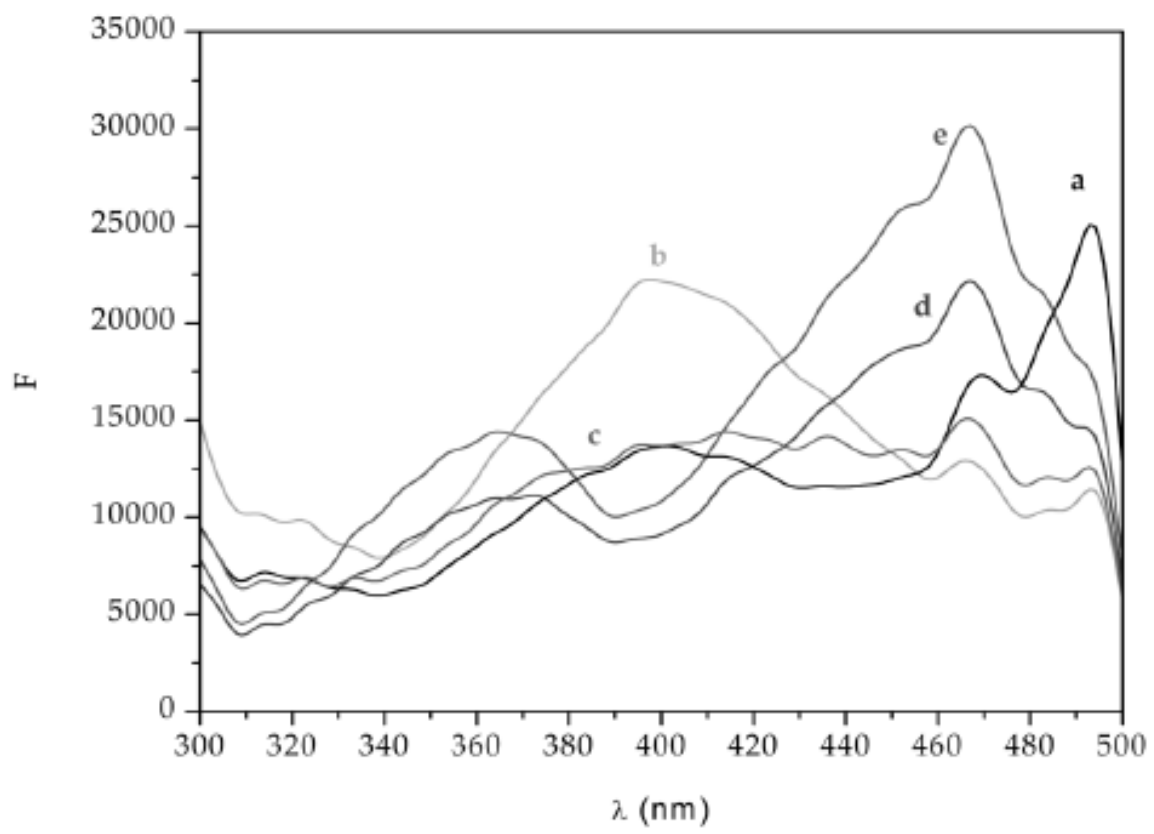

A

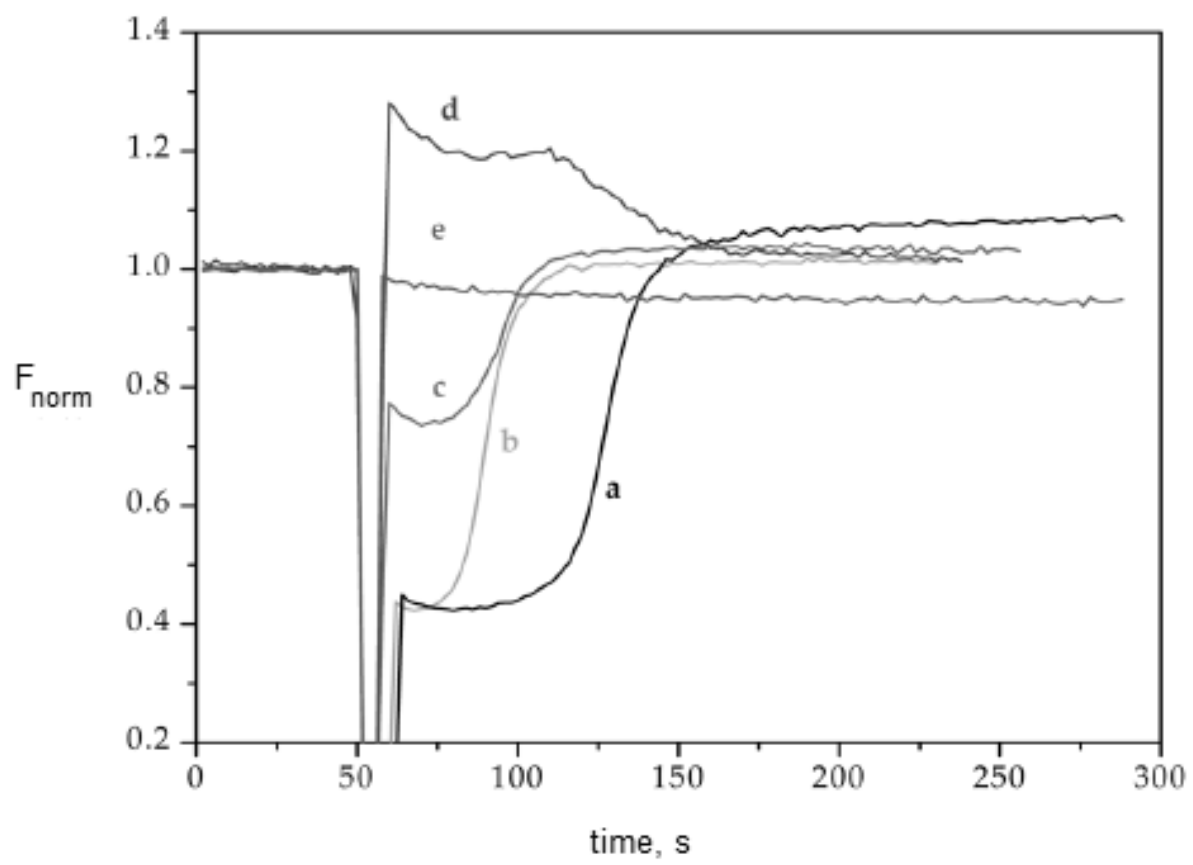

B 
Fig 5
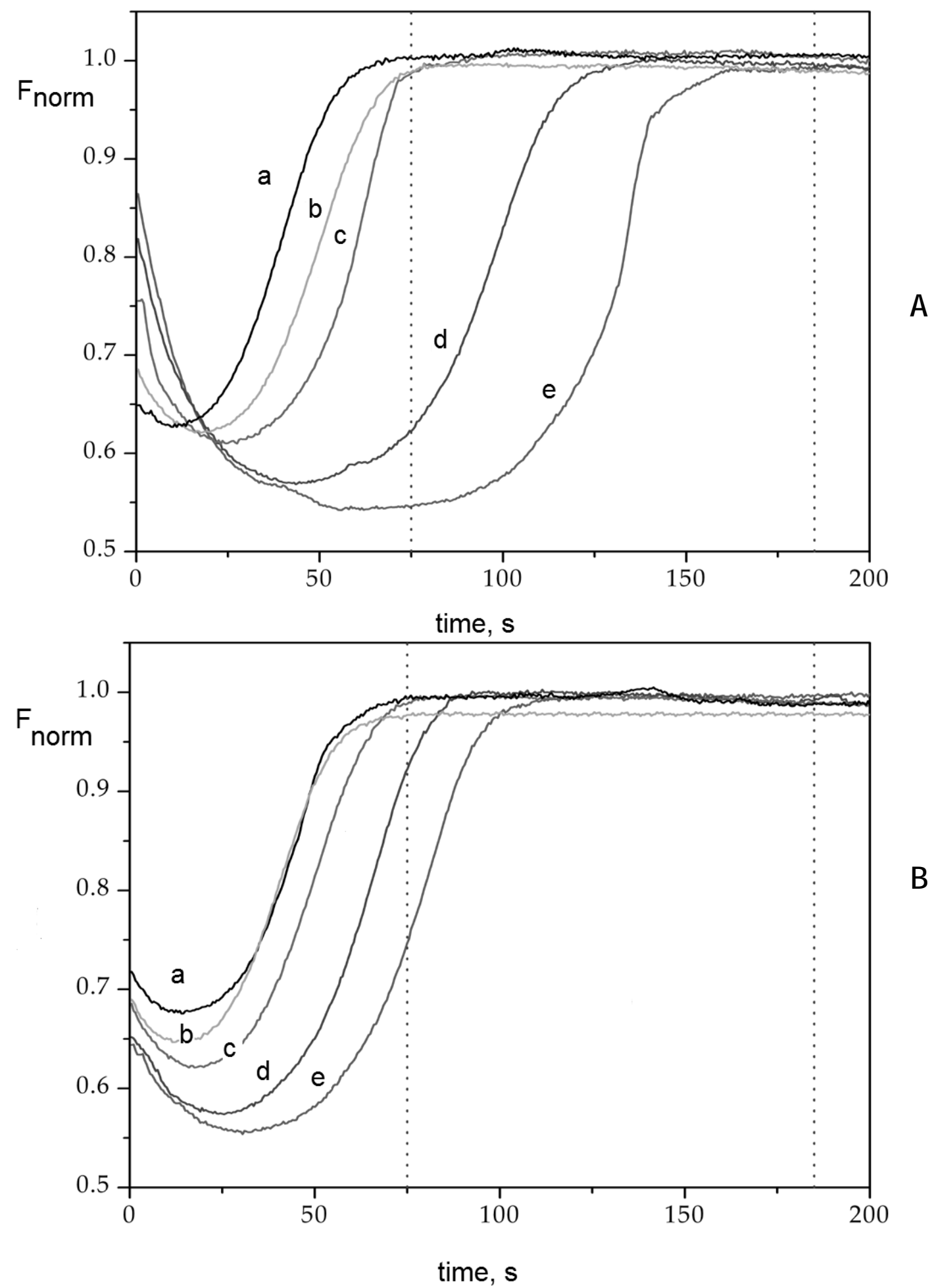